\title{
Índices de Castelli I e II como preditores robustos na estimativa do risco cardiovascular de adolescentes com excesso de peso
}

\author{
Castelli risk index I and II as robust predictors in estimating cardiovascular risk in excess weight \\ adolescents
}

Los índices de Castelli I y II como predictores robustos para estimar el riesgo cardiovascular en adolescentes con excesso de peso

\section{Resumo}

O presente estudo tem como objetivo avaliar a prevalência de dislipidemia e estimar o risco cardiovascular segundo indicadores bioquímicos mais precisos em adolescentes, de acordo com o estado nutricional. Trata-se de um estudo transversal realizado com 807 adolescentes de escolas públicas, de ambos os sexos, com idade entre 10 a 17 anos. $\mathrm{O}$ estado nutricional foi classificado de acordo com o índice de massa corporal. Foi considerada dislipidemia a presença de níveis aumentados em pelo menos um dos parâmetros do perfil lipídico: triglicerídeos (TG), colesterol total e frações de alta (HDL-c) e baixa densidade (LDL-c). O risco cardiovascular foi estimado pelo cálculo do Índice de Castelli I (IC-I) e II (IC-II), TG/HDL-c e não-HDL-c. Os dados foram analisados pela regressão de Poisson com estimativas robustas da variância com nível de significância de 5\%. A idade mediana foi de 13 (12-14) anos, dos quais $57,7 \%$ eram do sexo feminino. Foi encontrado uma prevalência de $32 \%$ de excesso de peso. O excesso de peso esteve associado ao risco cardiovascular em adolescentes, sendo o IC-II (RP: 2,75; IC95\%: 1,77-4,27; p<0,001) o marcador que teve maior prevalência no grupo excesso de peso, seguido do IC-I (RP: 1,87; IC95\%: 1,56-2,22; p<0,001). Foi possível concluir que, uso dos IC-I e II apresentaram prevalência superior aos marcadores lipídicos analisados isoladamente e, portanto, são preditores mais robustos do risco cardiovascular em adolescentes com excesso de peso. Palavras-chave: Índice; Adolescente; Doenças cardiovasculares; Sobrepeso; Obesidade.

\section{Abstract}

The present study aims to assess the prevalence of dyslipidemia and estimate cardiovascular risk according to more accurate biochemical indicators in adolescents, according to nutritional status. This is a cross-sectional study conducted with 807 adolescents from public schools, of both sexes, aged 10 to 17 years. The nutritional status was 
classified according to the body mass index. Dyslipidemia was considered the presence of increased levels in at least one of the parameters of the lipid profile: triglycerides (TG), total cholesterol and high (HDL-c) and low density (LDL-c) fractions. Cardiovascular risk was estimated by calculating Castelli Index I (CI-I) and II (CI-II), TG/HDL-c and non-HDL-c. Data were analyzed by Poisson regression with robust estimates of variance at $5 \%$ significance level. The median age was 13 (12-14) years, of which $57.7 \%$ were female. A $32 \%$ prevalence of excess weight was found. Excess weight was associated with cardiovascular risk in adolescents, and CI-II (PR: 2.75; 95\%CI: 1.77-4.27; $\mathrm{p}<0.001$ ) was the marker with the highest prevalence in the excess weight group, followed by CI-I (PR: 1.87; 95\%CI: 1.56-2.22; $\mathrm{p}<0.001)$. It was possible to conclude that the use of IC-I and II had a higher prevalence than lipid markers analyzed alone and, therefore, are more robust predictors of cardiovascular risk in excess weight adolescents.

Keywords: Index; Adolescents; Cardiovascular disease; Excess weight; Obesity.

\section{Resumen}

El presente estudio tiene como objetivo evaluar la prevalencia de dislipidemia y estimar el riesgo cardiovascular según indicadores bioquímicos más precisos en adolescentes, de acuerdo con el estado nutricional. Se trata de un estudio transversal realizado con 807 adolescentes de escuelas públicas, de ambos sexos, de entre 10 y 17 años. El estado nutricional se clasificó según el índice de masa corporal. Se consideró dislipidemia la presencia de niveles aumentados en al menos uno de los parámetros del perfil lipídico: triglicéridos (TG), colesterol total y fracciones de alta (HDL-c) y baja densidad (LDL-c). El riesgo cardiovascular se estimó mediante el cálculo del índice de Castelli I (CI-I) y II (CI-II), TG/HDL-c y no HDL-c. Los datos se analizaron mediante regresión de Poisson con estimaciones robustas de la varianza a un nivel de significación del 5\%. La edad media fue de 13 (12-14) años, de los cuales el $57,7 \%$ eran mujeres. Se encontró una prevalencia de exceso de peso del 32\%. El exceso de peso se asoció con el riesgo cardiovascular en los adolescentes, y el IC-II (PR: 2,75; IC 95\%: 1,77-4,27; p<0,001) fue el marcador con mayor prevalencia en el grupo con exceso de peso, seguido del IC-I (PR: 1,87; IC 95\%: 1,56-2,22; p<0,001). Se pudo concluir que el uso de CI-I y II tuvo una mayor prevalencia que los marcadores lipídicos analizados aisladamente y, por lo tanto, son predictores más robustos del riesgo cardiovascular en adolescentes con exceso de peso.

Palabras clave: Índice; Adolescentes; Enfermedad cardiovascular; Sobrepeso; Obesidad.

\section{Introdução}

As doenças cardiovasculares (DCV) são as principais causas de morte no mundo, representando $44 \%$ dos óbitos por doenças crônicas não transmissíveis (DCNT). Os principais fatores de risco para o desenvolvimento dessas doenças são relacionados aos hábitos de vida, com dietas desbalanceadas, sedentarismo, uso abusivo de álcool e de tabaco (World Health Organization, 2019). A obesidade é uma das principais consequências de um estilo de vida inadequado, apresentando forte relação com o desenvolvimento cada vez mais precoce de morbidades cardiovasculares e DCV, devido seu impacto negativo na pressão arterial, nos processos inflamatórios, diabetes e dislipidemias (DLP) (Arbués et al., 2019).

No mundo, a obesidade atinge 650 milhões de pessoas, sendo 340 milhões de crianças e adolescentes (World Health Organization, 2020). No Brasil, 25,5\% dos adolescentes de 12 a 17 anos estão com excesso de peso, apresentando uma prevalência de 8,4\% de obesidade (Bloch et al., 2016). Esses dados enfatizam a presença de fatores de risco cardiovascular (RCV) em indivíduos cada vez mais jovens, e esses reforçam o fenômeno de trilha, onde o processo aterosclerótico, preditor de DCV, iniciado na infância e adolescência se relaciona com eventos clínicos na vida adulta (Gidding et al., 2016).

As DLPs estão diretamente relacionadas ao surgimento da aterosclerose, que se caracteriza pelo acúmulo de partículas lipídicas nas camadas subendoteliais das artérias, acompanhado de resposta imune exacerbada, que favorece o desenvolvimento de lesões ateroscleróticas; condição essa agravada por modificações oxidativas ou não nas lipoproteínas (Lacy et al., 2019).

Nessa perspectiva, estudos brasileiros populacionais apontam que a prevalência de DLP entre crianças e adolescentes é de $10 \%$ a $35 \%$, variando entre as regiões e critérios diagnósticos utilizados para caracterizar a condição dislipidêmica (Simão et al., 2013). Isso reflete os hábitos inadequados de uma grande parcela da população brasileira, que apresenta elevada prevalência de tabagismo, tempo de tela, consumo excessivo de bebidas alcoólicas, baixo consumo de frutas e hortaliças, e inatividade física (Brasil, 2019). 
Apesar desses resultados, inexiste uma vigilância epidemiológica ao nível nacional que permita monitorar a saúde de adolescentes e a prevalência de DLP. Aliado a esse cenário e aos bem estabelecidos pontos de corte para lipoproteínas em adolescentes, a definição de risco cardiovascular nessa faixa etária ainda permanece amplamente controverso. Portanto, a aplicação de indicadores lipídicos associados ao tradicional perfil lipídico (colesterol total - CT, colesterol associado à lipoproteína de baixa densidade - LDL-c, colesterol associado à lipoproteína de alta densidade - HDL-c e triglicérides - TG) pode permitir o monitoramento mais preciso da saúde cardiovascular de adolescente. Sendo assim, alguns estudos têm testado o uso do dos Índices de Risco de Castelli I (IC-I, CT/HDL-c) e II (IC-II, LDL-c/HDL-c) (Kamoru et al., 2017) como estratégias para otimizar a estimativa do RCV em adolescentes.

Portanto, considerando a crescente prevalência de excesso de peso e obesidade entre adolescentes, torna-se importante a adoção de medidas mais precisas voltadas à avaliação e monitoramento do RCV nesta população. Neste estudo, nosso objetivo foi avaliar a prevalência de DLP e estimar o RCV, segundo indicadores bioquímicos mais precisos em adolescentes, de acordo com o estado nutricional.

\section{Metodologia}

Estudo epidemiológico, do tipo transversal, com abordagem descritiva e analítica realizado como parte de uma pesquisa maior intitulada "Indicadores do risco cardiometabólico em adolescentes de escolas municipais de Fortaleza Ceará". Foram incluídos adolescentes de 10 a 17 anos, de ambos os sexos, e matriculados em escolas municipais de ensino fundamental da cidade de Fortaleza-CE.

Para o cálculo amostral, fez-se a estratificação de acordo com as Secretarias Executivas Regionais (SER) de saúde. A população do estudo foi de 65.415 escolares matriculados no ano de 2015. Foi utilizada prevalência de $15 \%$ relativa aos estudantes com dislipidemias, erro amostral de 3\% e nível de significância de 5\%. A amostra estratificada foi de 775 acrescida de uma margem de $10 \%$ para possíveis perdas, resultando em uma amostra final de 853 estudantes adolescentes.

Excluíram-se aqueles que não estavam em jejum de 12 horas, adolescentes grávidas, bem como, os que referiram ter diagnóstico de diabetes, doença renal, doença hepática e doenças da tireoide. Os responsáveis e estudantes assinaram o Termo de Consentimento Livre e Esclarecido (TCLE) e Termo de Assentimento (TA), respectivamente. O estudo foi submetido e aprovado pelo Comitê de Ética do Hospital Infantil Albert Sabin, sob parecer n ${ }^{\circ} .327851$, em conformidade com a Resolução 466/2012.

Por meio da aplicação de um questionário estruturado e realização de entrevista direta foram obtidas informações sobre sexo, idade e data de nascimento. As medidas antropométricas dos adolescentes foram aferidas por nutricionistas, conforme preconizado por Lohman (1986). A altura foi aferida com auxílio de um estadiômetro portátil da marca Sanny®, com amplitude de $200 \mathrm{~cm}$ e variação de $0,1 \mathrm{~cm}$, e os adolescentes encostando a panturrilha e região occipital no aparelho e a leitura realizada, com precisão de $0,1 \mathrm{~cm}$. O peso foi aferido em balança eletrônica portátil Seca®, com capacidade de $150 \mathrm{~kg}$ e precisão de $0,1 \mathrm{~kg}$.

Para a definição do estado nutricional foi calculado o Índice de Massa Corporal (IMC) e classificado a partir do Percentil de IMC por idade, conforme orientações do Sistema de Vigilância Alimentar e Nutricional (SISVAN) (Brasil, 2011), sendo categorizados em: magreza (Percentil <3), eutrofia (Percentil $\geq 3$ e $<85$ ), sobrepeso (Percentil $\geq 85$ e $<97$ ) e obesidade (Percentil $\geq 97$ ).

A coleta de sangue para as análises bioquímicas foi realizada após 12 a 14 horas de jejum, sendo o sangue coletado em tubos com EDTA $(1,0 \mathrm{mg} / \mathrm{mL})$. A partir do plasma obtido por meio de centrifugação a $3000 \mathrm{rpm}$, a $4{ }^{\circ} \mathrm{C}$ e por 15 minutos foram realizadas as análises do colesterol total (CT), colesterol associado à HDL (HDL-c) e triglicerídeos (TG). A 
concentração de colesterol associado à LDL (LDL-c) foi calculada a partir da equação de Friedwald, Levy e Fredrickson (1972).

Foram utilizados métodos padrão e kits comerciais (Roche Diagnosis®) aplicados em sistema automatizado (Roche Diagnosis $($ ). Todas as análises foram realizadas em duplicata, sendo a variabilidade intra e inter análises, menor que $15 \%$ para todos os parâmetros. As análises foram realizadas no laboratório de análises clínicas LEG (Santa Casa de Misericórdia de Fortaleza, Ceará).

Os adolescentes foram considerados dislipidêmicos quando houve presença de CT ( $\geq 170 \mathrm{mg} / \mathrm{dL})$, LDL-c $(\geq 110$ $\mathrm{mg} / \mathrm{dL})$ ou TG ( $\geq 90 \mathrm{mg} / \mathrm{dL})$, ou baixo HDL-c $(\leq 45 \mathrm{mg} / \mathrm{dL})$, conforme proposto pela Atualização da Diretriz Brasileira de Dislipidemias e Prevenção da Aterosclerose (Faludi et al., 2017). Além da presença da DLP, o RCV foi estimado pelo cálculo do Índice de Castelli I (CT/ HDL-c), Índice de Castelli II (LDL-c/HDL-c), TG/HDL-c e não-HDL (CT-HDL-c).

As variáveis categóricas foram descritas em frequências absolutas e relativas, e as contínuas, em mediana e intervalo interquartil. Após verificação da normalidade dos dados (teste Kolmogorov-Smirnov) e homogeneidade (teste de Levene) foram feitas comparações entre as variáveis de interesse em relação ao sexo, utilizando o teste Mann-Whitney e as diferenças estatísticas foram avaliadas usando o teste de Qui-quadrado de Pearson para variáveis categóricas (SPSS versão 20.0).

A análise de variância entre o estado nutricional e as variáveis de interesse (CT, LDL-c, HDL-c, TG, IC-I, IC-I, TG/HDL-c e Não-HDL-c) foram realizadas no programa GraphPad Prism 8, sendo utilizado o teste Kruskal-Wallis seguido de pós-teste Dunn. Devido ao número de indivíduos, as variáveis magreza e eutrofia foram agrupadas para melhor expressão dos resultados.

Para avaliar a associação univariada e múltipla entre o estado nutricional e o risco cardiovascular, foi realizada a regressão de Poisson com variâncias robustas para estimar a razão de prevalência (RP) e os respectivos intervalos de confiança de 95\% (IC 95\%), tendo como variáveis de ajuste o sexo e a idade. O estado nutricional foi analisado como variável dicotômica: sem excesso de peso (magreza e eutrofia) e com excesso de peso (sobrepeso e obesidade), enquanto o risco cardiovascular foi obtido pela frequência de dislipidemias e índices (IC-I e II, TG/HDL-c, Não-HDL-c). Devido à ausência de pontos de corte específicos para adolescentes, foram utilizados os valores propostos para adultos, sendo considerados normais: IC-I $<3,5$ (Arcanjo et al., 2005) e IC-II $\leq 2,9$ (Brandão et al., 2015). Para TG/HDL e não-HDL, a mediana dos valores encontrados foi usada como ponto de corte. Em todas as análises o nível de significância estatística foi de 5\%.

\section{Resultados}

Após aplicação dos critérios de exclusão, o tamanho amostral foi definido em 807 adolescentes (Figura 1), dos quais $57,7 \%$ eram do sexo feminino com mediana de idade de 13 (IIQ = 12-14) anos. Houve diferença significativa entre os sexos em relação à altura, CT, HDL-c, LDL-c e TG. Em relação ao estado nutricional, a maioria dos adolescentes estava com peso adequado, entretanto observou-se que 32\% apresentavam excesso de peso. Dos índices de RCV avaliados, somente o NãoHDL-c apresentou diferença significativa entre os sexos, com maior mediana e maior número de indivíduos com valores acima do ponto de corte no sexo feminino ( $\mathrm{p}=0,004 \mathrm{e} \mathrm{p}=0,002$, respectivamente) (Tabela 1). 
Figura 1. Fluxograma amostral do estudo.

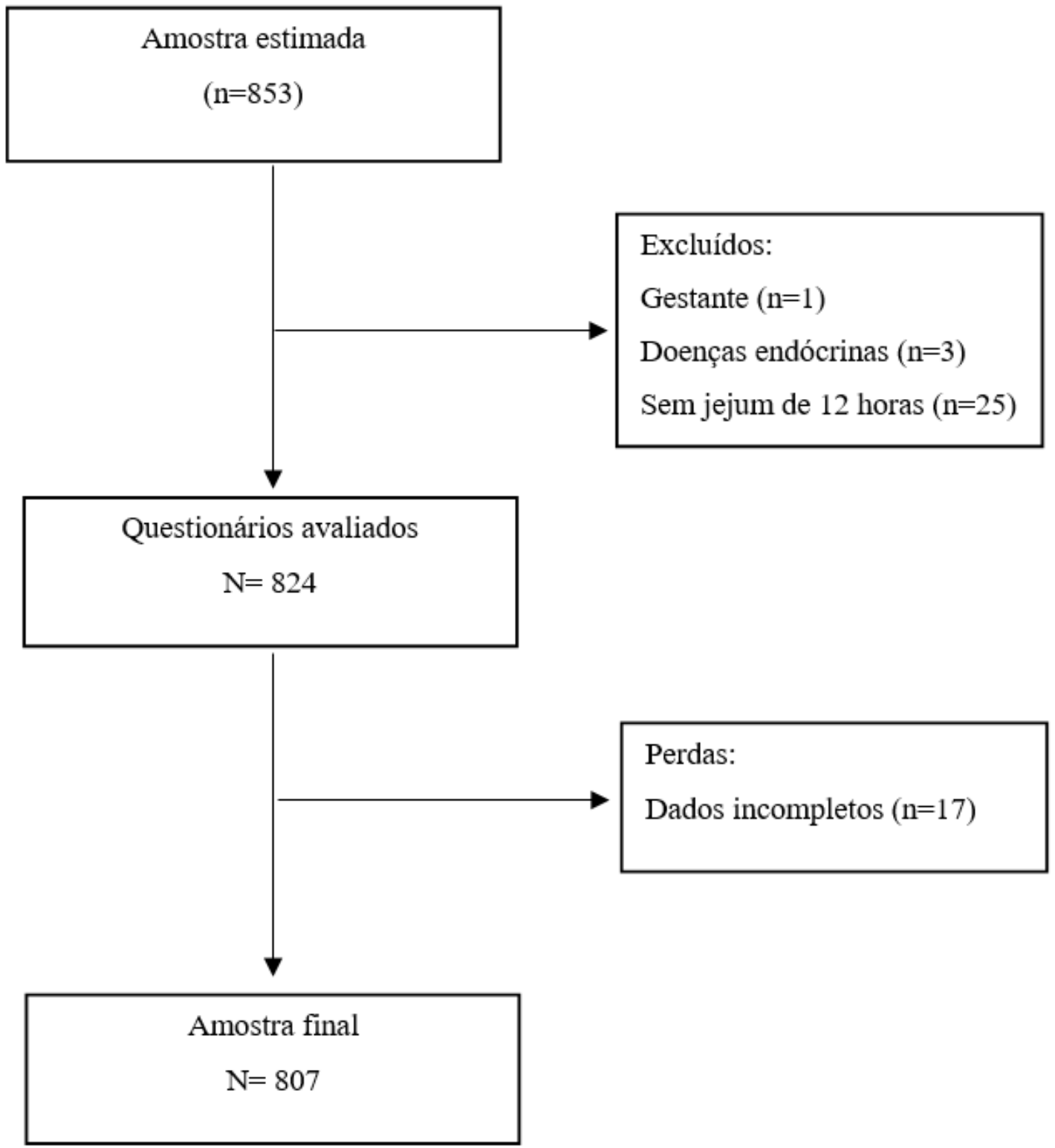

Fonte: Autores. 
Tabela 1. Características dos adolescentes, dados antropométricos e perfil lipídico, de acordo com o sexo, Fortaleza, Brasil

\begin{tabular}{|c|c|c|c|c|}
\hline Variáveis & Total $n=807$ & Masculino $n=343$ & Feminino $n=464$ & Valor de $\mathrm{p}^{*}$ \\
\hline Idade, anos, mediana (IIQ) & $13(12-14)$ & $13(12-14)$ & $13(12-14)$ & 0,142 \\
\hline Peso, kg, mediana (IIQ) & $48,2(41,7-57,2)$ & $48,3(40,4-58,7)$ & $48,2(42,4-55,6)$ & 0,543 \\
\hline Altura, m, mediana (IIQ) & $1,55(1,50-1,61)$ & $1,56(1,49-1,64)$ & $1,55(1,50-1,59)$ & 0,001 \\
\hline IMC, kg/m2, mediana (IIQ) & $19,8(17,9-22,6)$ & $19,3(17,6-22,4)$ & $20(18,1-22,7)$ & 0,096 \\
\hline \multicolumn{5}{|l|}{ Estado Nutricional, n (\%) } \\
\hline magreza/eutrofia & $549(68 \%)$ & $222(64,7 \%)$ & $327(70,5 \%)$ & \\
\hline sobrepeso & $145(18 \%)$ & $62(18,1 \%)$ & $83(17,9 \%)$ & 0,070 \\
\hline obesidade & $113(14 \%)$ & $59(17,2 \%)$ & $54(11,6 \%)$ & \\
\hline CT, mg/dL, mediana (IIQ) & $145,3(129,5-162)$ & $142,7(124,5-156,3)$ & $149,1(133,7-164,9)$ & $<0,001$ \\
\hline HDL-c, mg/dL, mediana (IIQ) & $45,3(38,5-52,3)$ & $44(37,7-50,9)$ & $45,8(39,5-5,3)$ & 0,008 \\
\hline LDL-c, mg/dL, mediana (IIQ) & $83,2(70,7-98)$ & $81,9(68,4-95,7)$ & $84(71,6-99,1)$ & 0,048 \\
\hline TG, mg/dL, mediana (IIQ) & $73,5(56,1-99)$ & $69(53,5-93)$ & $78,4(57,7-101)$ & $\mathbf{0 , 0 0 3}$ \\
\hline DLP, sim, n (\%) & $529(65,5 \%)$ & $220(64,1 \%)$ & $309(66,6 \%)$ & 0,468 \\
\hline IC-I, mediana (IIQ) & $3,18(2,72-3,82)$ & $3,16(2,71-3,88)$ & $3,21(2,74-3,79)$ & 0,918 \\
\hline IC-I, $\geq 3,5, n(\%)$ & $302(37,4 \%)$ & $132(38,5 \%)$ & $170(36,6 \%)$ & 0,592 \\
\hline IC-II, mediana (IIQ) & $1,84(1,49-2,34)$ & $1,83(1,49-2,36)$ & $1,86(1,46-2,32)$ & 0,596 \\
\hline IC-II, >2,9, n (\%) & $76(9,4 \%)$ & $37(10,8 \%)$ & $39(8,4 \%)$ & 0,252 \\
\hline TG/HDL, mediana (IIQ) & $1,63(1,16-2,4)$ & $1,55(1,11-2,39)$ & $1,67(1,19-2,4)$ & 0,242 \\
\hline $\mathrm{TG} / \mathrm{HDL}>1,63, \mathrm{n}(\%)$ & $405(50,2 \%)$ & $161(46,9 \%)$ & $244(52,6 \%)$ & 0,113 \\
\hline Não-HDL, mediana (IIQ) & $99,7(85,5-116)$ & $96,2(80,1-114)$ & $102,2(88-118,2)$ & 0,004 \\
\hline Não-HDL >99,7, n (\%) & $403(49,9 \%)$ & $150(43,7 \%)$ & $253(54,5 \%)$ & $\mathbf{0 , 0 0 2}$ \\
\hline
\end{tabular}

Valores descritos em mediana (IIQ) ou n (\%); IMC: índice de massa corporal; CT: colesterol total; HDL-c: colesterol associado à lipoproteína de alta densidade; LDL-c: colesterol associado à lipoproteína de baixa densidade; TG: triglicerídeos; DLP: dislipidemias; IC-I: índice de Castelli-I; IC-II: índice de Castelli-II; RCV: risco cardiovascular; *teste Mann Whitney para comparar variáveis contínuas e teste de Qui-quadrado de Pearson para comparar variáveis categóricas; significância estatística p<0,05. Fonte: Autores.

Considerando o perfil lipídico e os índices de RCV, as médias de CT e HDL-c, tiveram diferenças significativas entre os grupos Magreza/Eutrofia e Obesidade, sendo o CT maior e o HDL-c menor nos adolescentes obesos (Figura 2A e 2C). LDL-c e TG tiveram médias significativamente diferentes entre todos os grupos avaliados, apresentando um aumento nos valores proporcional ao incremento no IMC (Figura 2B e 2D).

RCV estimado pelos IC-I e -II, TG/HDL-c e Não-HDL-c aumentou de acordo com piora do estado nutricional, mostrando diferença significativa entre todos os grupos avaliados (Figura 3). 
Figura 2. Associação entre os parâmetros relacionados à dislipidemia e o estado nutricional dos adolescentes.

A

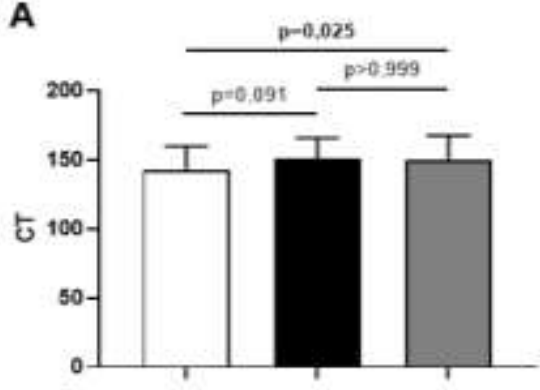

C

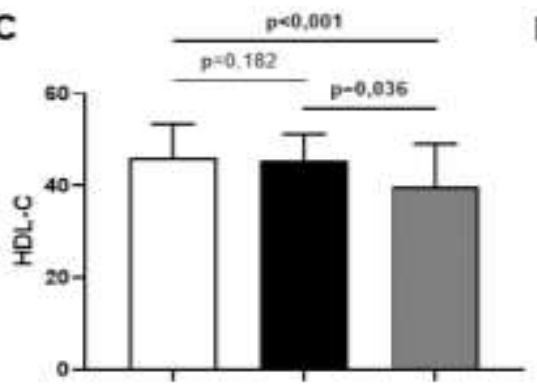

B

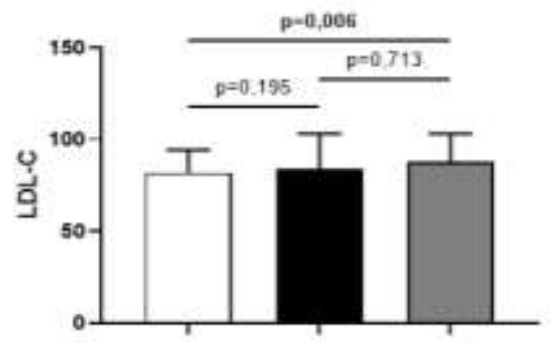

D

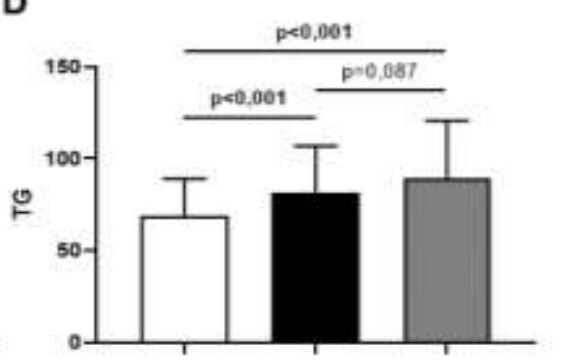

$\square$ Magreza/Eutrofia

- Sobrepeso

$\square$ Obesidade

A) CT, colesterol total. B) LDL-c, colesterol associado à lipoproteína de baixa densidade. C) HDL-c, colesterol associado à lipoproteína de alta densidade. D) TG, triglicerídeos. Comparação entre grupos foi realizada pelo Kruskal-Wallis e pós-teste de Dunn. Significância estatística $\mathrm{p}<0,05$. Fonte: Autores.

Figura 3. Associação entre os índices preditores de risco cardiovascular e o estado nutricional dos adolescentes.

A

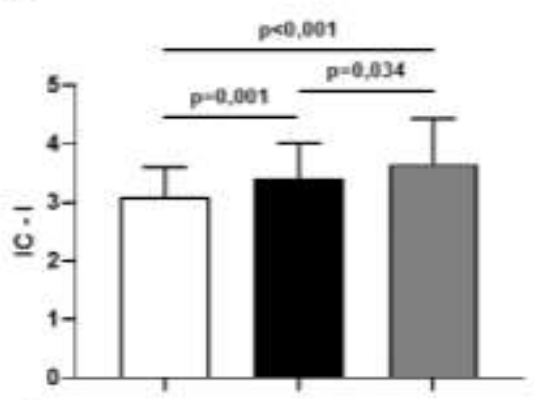

C

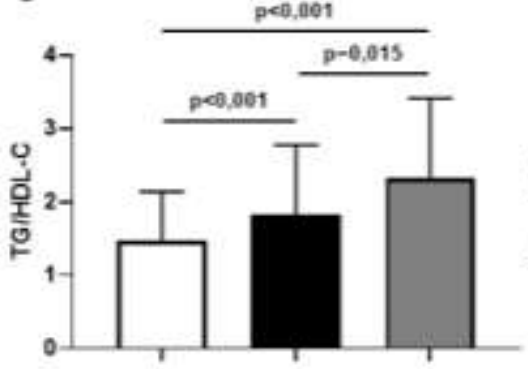

B

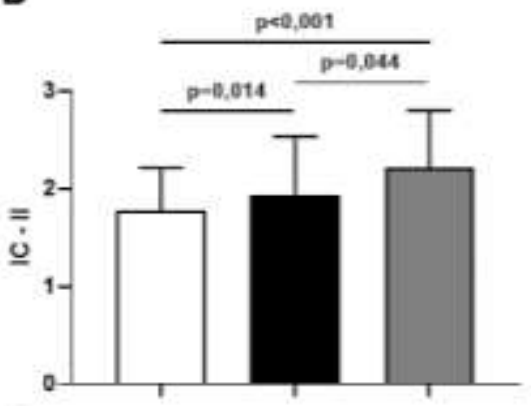

D

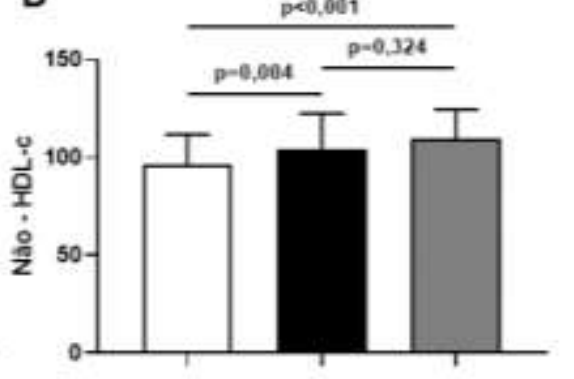

口 MagrezaEutrofia

- Sobrepeso

口 Obesidade

A) IC-I, índice de Castelli I. B) IC-II, índice de Castelli II. C) TG/HDL-c, triglicerídeos/colesterol associado à lipoproteína de alta densidade. D) Não-HDL-c, não- colesterol associado à lipoproteína de alta densidade. Comparação entre grupos foi realizada pelo Kruskal-Wallis e pósteste de Dunn. Significância estatística p<0,05. Fonte: Autores. 
$\mathrm{Na}$ análise múltipla, o excesso de peso esteve associado diretamente ao RCV em adolescentes, sendo o IC-II o marcador que teve maior prevalência no grupo excesso de peso, seguido do IC-I. O excesso de peso aumentou em 175\% e 87\% o RCV, segundo esses índices, respectivamente. Além disso, a dislipidemia, os índices TG/HDL-c e Não-HDL-c estiveram associados diretamente ao excesso de peso $(\mathrm{p}<0,05)$. A razão de prevalência entre biomarcadores e índices lipídicos se manteve significativa após ajuste pela idade e sexo, indicando a forte influência do estado nutricional no RCV de adolescentes em ambos os sexos e em todas as idades (Figura 4).

Figura 4. Razão de prevalência do risco cardiovascular, predito pelos índices avaliados, em adolescentes com excesso de peso.

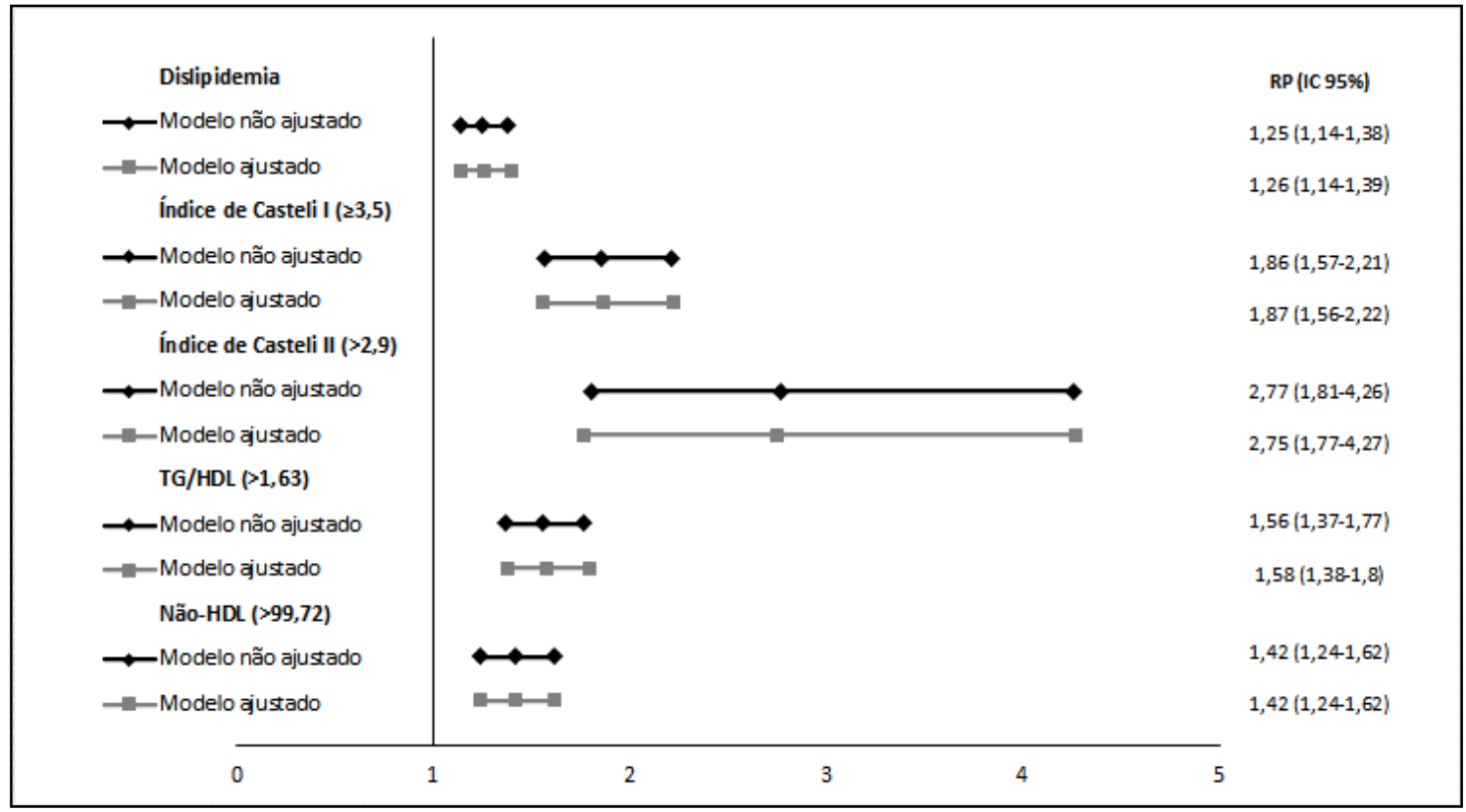

TG/HDL: triglicerídeos/colesterol associado à lipoproteína de alta densidade. Não-HDL-c, colesterol não associado à lipoproteína de alta densidade. RP: Regressão de Poisson (razão de prevalência); IC: intervalo de confiança de 95\%. Modelo ajustado por sexo e idade. Teste Qui-quadrado de Wald: *p<0,001. Fonte: Autores.

\section{Discussão}

Os resultados desse estudo mostraram que os IC-I e IC-II são marcadores mais prevalentes em adolescentes com excesso de peso que os tradicionais parâmetros lipídicos analisados isoladamente, em ambos os sexos e em todas as idades. Esses resultados não excluem a importância do monitoramento anual do perfil lipídico tradicional entre os adolescentes, mas reforçam a relevância de incluir os IC-I e -II devido sua maior precisão.

O uso dos IC-I e IC-II têm sido descritos na literatura como potencial preditor de RCV e eventos cardiovasculares na população adulta, principalmente na presença de doenças crônicas (Gowda et al., 2019; Feoli et al., 2018; Kamoru et al., 2017). Na população infanto juvenil, os IC-I e -II já foram utilizados na caracterização do perfil lipídico de escolares no Rio de Janeiro (Scherr, Magalhães, \& Malheiros, 2007), na avaliação de intervenções para melhoria no estilo de vida em escolares de Santa Catarina (Rosini et al., 2014) e em estudo sobre síndrome metabólica em crianças de São Paulo (Barbalho et al., 2017). Nossos resultados acrescentam informação sobre a relevância desses índices no monitoramento do RCV em adolescentes, principalmente em grupos com excesso de peso, uma vez que ainda são poucos os estudos utilizando esses índices nessa população.

De fato, o CT, assim como o LDL-c e os TG apresentaram valores superiores e diretamente proporcionais ao IMC nos adolescentes estudados. Esses resultados mostram que o sobrepeso e a obesidade durante esse período da vida exercem impacto negativo significativo no risco precoce de desenvolvimento de outros fatores de risco e doenças coronarianas. Barros 
et al. (2020) concluíram por meio de uma revisão integrativa, que crianças e adolescente com peso adequado tinham, em sua maioria, perfil lipídico normal, entretanto, os que tinham excesso de peso apresentaram níveis mais elevados de CT, LDL-c, TG e níveis menores de HDL-c, caracterizando a presença de DLP nesses indivíduos.

A alta prevalência de DLP em adolescentes, principalmente no Norte e Nordeste do Brasil, também foi descrita no Estudo de Riscos Cardiovasculares em Adolescentes (ERICA) (Faria Neto et al., 2016), caracterizado principalmente por hipertrigliceridemia e baixa concentração de HDL-c. Vizentin et al. (2019) encontraram, além da alta prevalência de DLP, associação significativa entre elevada concentração de TG, baixo conteúdo de HDL-c e sobrepeso em adolescentes. Em nosso estudo, a hipertrigliceridemia entre os adolescentes obesos foi $30 \%$ superior à observada entre os indivíduos do grupo magreza/eutrofia, enquanto que o conteúdo de colesterol associado à HDL foi $14 \%$ inferior.

O aumento na concentração de triglicerídeos plasmáticos é visto como um sinal de risco aumentado para DCV por mecanismos envolvendo a disfunção endotelial, inflamação e regulação de citocinas pró-inflamatórias que favorecem o processo de aterogênese (Peng et al., 2017). Embora nosso estudo não tenha investigado o consumo alimentar dos adolescentes, sabe-se que o binômio hipertrigliceridemia-hipoHDL-c é reflexo direto de uma dieta rica em carboidratos refinados e açúcares, em detrimento do baixo consumo de frutas e legumes (dos Santos Vaz et al., 2018).

De acordo com Kavey (2015), essas alterações lipídicas estão inseridas no padrão altamente aterogênico denominado dislipidemia combinada, cuja prevalência entre adolescentes obesos é de 40\%. Sabendo-se ainda que o HDL-c tem função cardioprotetora, associada ao transporte reverso de colesterol, diminuindo o acúmulo de colesterol nos tecidos periféricos, favorecendo a excreção biliar do excesso colesterol nas fezes via ativação da enzima 7alfa-hidroxilase ao nível hepático (Wang et al., 2017).

Mais recentemente, um estudo avaliando o excesso de peso, o perfil lipídico e a qualidade da dieta de adolescente, mostrou que o grupo com IMC maior apresentou alterações significativas no perfil lipídico e glicídico, comparado ao grupo eutrófico, e $60 \%$ de todos os adolescentes estudados tinham uma dieta de baixa qualidade (Çağiran Yilmaz, Çağiran, \& Özçelik, 2019). Reuter et al. (2019), estudaram crianças e adolescentes do sul do Brasil e encontraram maiores chances de hipercolesterolemia e hipertrigliceridemia no grupo com excesso de peso.

A faixa etária que abrange a adolescência é a que menos obedece às recomendações alimentares, apresentando baixa qualidade da dieta, geralmente derivada de alto consumo de ácidos graxos saturados em comparação aos ácidos graxos insaturados, baixo consumo de componentes saudáveis, como frutas, verduras e grãos integrais, consequentemente levando a um inadequado consumo de fibras alimentares (Ruiz et al., 2020). O consumo de gorduras saturadas superior a $10 \%$ foi associado ao maior risco de ter concentrações baixas de HDL-c no Nordeste (Carvalho et al., 2020).

A hipertrofia e a hiperplasia do tecido adiposo fazem parte do processo fisiológico de maturação na adolescência, entretanto quando associadas à obesidade leva ao aumento na secreção de adipocinas pró-inflamatórias, alterando diversos mecanismos fisiológicos, que favorecem à resistência à insulina (RI) e disfunções no metabolismo lipídico (Vekic et al., 2019). A prevalência de excesso de peso encontrada neste estudo (32\%) foi relativamente alta quando comparada a descrita no Nordeste, segundo o ERICA (24,2\%) (Bloch et al., 2016). Esse fato pode explicar a maior prevalência de DLP e RCV estimado pelos IC-I e -II nos adolescentes com excesso de peso no presente estudo.

Embora a classificação do estado nutricional com base no IMC possa apresentar limitações, o estudo de Sheibani et al. (2019), comparou o IMC com o percentual de gordura corporal como preditores de DCV. De acordo com esses autores o IMC apresentou melhor poder preditor para hipertensão arterial, DM e DLP.

Este estudo possui limitações. Apesar dos resultados mostrarem a superioridade dos IC-I e IC-II sobre os marcadores lipídicos tradicionais, consideramos o número de parâmetros bioquímicos avaliados modesto e, portanto, nossos resultados não podem ser extrapolados a outros novos marcadores e índices cardiometabólicos descritos na literatura. O tamanho amostral foi 
considerado estatisticamente representativo, entretanto, o universo de adolescentes no Brasil é muito superior, além de estar submetido a outros determinantes que impactam no metabolismo lipídico, como o nível de atividade física. Desse modo, nossos resultados devem ser confirmados em outros grupos populacionais.

Apesar de não haver pontos de corte estabelecidos para adolescentes, os IC-I e -II se mostraram preditores de RCV mais precisos do que frações lipídicas analisadas isoladas. Além da facilidade de aplicação e baixo custo dos IC-I e -II, a análise do balanço entre lipoproteínas aterogênicas e cardioprotetoras traz um olhar mais preciso sobre o atual RCV dos adolescentes, assim como pode subsidiar de modo mais preciso o impacto de intervenções farmacológicas e de estilo de vida (Belalcazar et al., 2020; Bhardwaj et al., 2013).

\section{Conclusão}

Nossos resultados mostraram que adolescentes com sobrepeso e obesidade apresentam diversos parâmetros lipídicos alterados (CT, LDL-c, TG e HDL-c). O uso dos IC-I e -II apresentaram prevalência superior aos marcadores lipídicos analisados isoladamente, independente de sexo e idade. Portanto, os IC-I e -II são preditores mais robustos do RCV em adolescentes. Os resultados apresentados nesse estudo alertam para o monitoramento precoce de fatores de RCV no público pediátrico.

\section{Agradecimentos}

Ao Grupo de Estudo e Pesquisa em Micronutrientes e Doenças Crônicas (GMIC) da Universidade Estadual do Ceará.

\section{Referências}

Arbués, E. R., Martínez-Abadía, B., Gracía-Tabuenca, T., Yuste-Gran, C., Pellicer-García, B., Juárez-Vela, R., \& Sáez-Guinoa, M. (2019). Prevalencia de sobrepeso/obesidad y su asociación con diabetes, hipertensión, dislipemia y síndrome metabólico: estudio transversal de una muestra de trabajadores en Aragón, España. Nutrición Hospitalaria, 36(1), 51-59.

Arcanjo, C. L., Piccirillo, L. J., Machado, I. D. V., de Andrade Jr, C. R., Clemente, E. L., \& Gomes, M. D. B. (2005). Avaliação de dislipidemia e de índices antropométricos em pacientes com diabetes mellitus tipo 1. Arquivos Brasileiros de Endocrinologia \& Metabologia, 49(6), 951-958.

Barbalho, S. M., Oshiiwa, M., Fontana, L. C. S., Finalli, E. F. R., Paiva Filho, M. E., \& Spada, A. P. M. (2017). Metabolic syndrome and atherogenic indices in school children: A worrying panorama in Brazil. Diabetes \& Metabolic Syndrome: Clinical Research \& Reviews, 11 , S397-S401.

Barros, A. M. G., Visco, D. B., da Silva, R. M. P., da Paiva Alves, S. P., da Silva Simões, M. O., Medeiros, C. C. M., \& de Carvalho, D. F. (2020). Perfil lipídico em crianças com sobrepeso e obesidade: uma revisão integrativa. Research, Society and Development, 9(11), e4349119952-e4349119952.

Bhardwaj, S., Bhattacharjee, J., Bhatnagar, M. K., Tyagi, S., \& Delhi, N. (2013). Atherogenic index of plasma, castelli risk index and atherogenic coefficientnew parameters in assessing cardiovascular risk. Int J Pharm Biol Sci, 3(3), 359-64.

Bloch, K. V., Klein, C. H., Szklo, M., Kuschnir, M. C. C., Abreu, G. D. A., Barufaldi, L. A., \& Goldberg, T. B. L. (2016). ERICA: prevalências de hipertensão arterial e obesidade em adolescentes brasileiros. Revista de Saúde Pública, 50, 9s.

Brandão, J. M., Fernandes, C. D. S., Barroso, S. G., \& Rocha, G. D. S. (2015). Associação do consumo de fibras e risco cardiovascular em pacientes idosos. International Journal of Cardiovascular Sciences, 28(6), 464-71.

Brasil. Ministério da Saúde. (2011). Secretaria de Atenção à Saúde. Orientações para a coleta e análise de dados antropométricos em serviços de saúde: norma técnica do sistema de vigilância alimentar e nutricional - SISVAN. Brasília: Ministério da Saúde. http://189.28.128.100/dab/docs/portaldab/publicacoes/orientacoes_coleta_analise_dados_antropometricos.pdf

Brasil. Ministério da Saúde. (2019). Vigitel Brasil 2018: vigilância de fatores de risco e proteção para doenças crônicas por inquérito telefônico: estimativas sobre frequência e distribuição sociodemográfica de fatores de risco e proteção para doenças crônicas nas capitais dos 26 estados brasileiros e no Distrito Federal em 2018. Brasília: Ministério da Saúde. https://portalarquivos2.saude.gov.br/images/pdf/2019/julho/25/vigitel-brasil 2018.pdf

Çağiran Yilmaz, F., Çağiran, D., \& Özçelik, A. Ö. (2019). Adolescent obesity and its association with diet quality and cardiovascular risk factors. Ecology of food and nutrition, 58(3), 207-218.

Carvalho, L., Santos, M., Cabral, S., Oliveira, V., \& Lopes, T. (2020). ERICA: Consumption of trans fats and saturated fats associated with dyslipidemia in obese and overweight adolescents. Rev. chil. nutr, 73-79.

dos Santos Vaz, J., Buffarini, R., Kac, G., Bielemann, R. M., Oliveira, I., Menezes, A. B., \& Assunção, M. C. F. (2018). Dietary patterns are associated with blood lipids at 18-year-olds: a cross-sectional analysis nested in the 1993 Pelotas (Brazil) birth cohort. Nutrition journal, 17(1), 1-12. 
Faludi, A. A., Izar, M. C. D. O., Saraiva, J. F. K., Chacra, A. P. M., Bianco, H. T., Afiune Neto, A., \& Salgado Filho, W. (2017). Atualização da diretriz brasileira de dislipidemias e prevenção da aterosclerose-2017. Arquivos brasileiros de cardiologia, 109(2), 1-76.

Faria Neto, J. R., Bento, V. F. R., Baena, C. P., Olandoski, M., Gonçalves, L. G. D. O., Abreu, G. D. A., \& Bloch, K. V. (2016). ERICA: prevalência de dislipidemia em adolescentes brasileiros. Revista de Saúde Pública, 50,10s.

Feoli, A. M. P., Ribeiro, É. C. T., Piovesan, C. H., Macagnan, F. E., da Silva Oliveira, M., \& da Silva Gustavo, A. (2018). Melhora do estilo de vida reduz o índice de Castelli 1 em indivíduos com síndrome metabólica. Revista Saúde e Pesquisa. 11(3):467-74.

Friedewald, W. T., Levy, R. I., \& Fredrickson, D. S. (1972). Estimation of the concentration of low-density lipoprotein cholesterol in plasma, without use of the preparative ultracentrifuge. Clinical chemistry, 18(6), 499-502.

Gidding, S. S., Rana, J. S., Prendergast, C., McGill, H., Carr, J. J., Liu, K., \& McMahan, C. A. (2016). Pathobiological determinants of atherosclerosis in youth (PDAY) risk score in young adults predicts coronary artery and abdominal aorta calcium in middle age: the CARDIA study. Circulation, 133(2), 139-146.

Gowda, Y. S., Asha Rani, N., Rajeshwari, A., Somashekar, G. N., \& Nusrath, A. (2019) Atherogenic Predictor Indices: Role in Assessment of Cardiovascular Risk in Type 2 Diabetes Mellitus. IOSR J Biotechnol Biochem. 5(4):36-41.

Kamoru, A. A., Japhet, O. M., Adetunji, A. D., Musa, M. A., Hammed, O. O., Akinlawon, A. A., ... \& Roji, S. M. (2017). Castelli risk index, atherogenic index of plasma, and atherogenic coefficient: emerging risk predictors of cardiovascular disease in hiv-treated patients. Saudi Journal of Medical and Pharmaceutical Sciences, 1101-1110.

Kavey, R. E. W. (2015). Combined dyslipidemia in childhood. Journal of clinical lipidology, 9(5), S41-S56.

Lacy, M., Atzler, D., Liu, R., de Winther, M., Weber, C., \& Lutgens, E. (2019). Interactions between dyslipidemia and the immune system and their relevance as putative therapeutic targets in atherosclerosis. Pharmacology \& therapeutics, 193, 50-62.

Lohman, T. G. (1986). Applicability of body composition techniques and constants for children and youths. Exercise and sport sciences reviews, $14,325-357$.

Peng, J., Luo, F., Ruan, G., Peng, R., \& Li, X. (2017). Hypertriglyceridemia and atherosclerosis. Lipids in health and disease, 16(1), 1-12.

Reuter, C. P., Brand, C., Silva, P. T. D., Reuter, É. M., Renner, J. D. P., Franke, S. I. R., \& Burgos, M. S. (2019). Relação entre Dislipidemia, Fatores Culturais e Aptidão Cardiorrespiratória em Escolares. Arquivos Brasileiros de Cardiologia, 112(6), 729-736.

Rosini, N., Rosini, R., Bruns, E., Camillo, G., Machado, M., \& Silva, E. (2014). Jogos recreativos melhoram os fatores de risco cardiovascular em crianças e adolescentes com dislipidemia e obesidade abdominal: Um estudo piloto. Revista Brasileira de Atividade Física \& Saúde, 19(1), 121-121.

Ruiz, L. D., Zuelch, M. L., Dimitratos, S. M., \& Scherr, R. E. (2020). Adolescent obesity: diet quality, psychosocial health, and cardiometabolic risk factors. Nutrients, 12(1), 43 .

Salcedo-Cifuentes, M., Belalcazar, S., Acosta, E. Y., \& Medina-Murillo, J. J. (2020). Conventional biomarkers for cardiovascular risks and their correlation with the Castelli Risk Index-Indices and TG/HDL-C. Archivos de Medicina (Manizales), 20(1), 11-22.

Scherr, C., Magalhães, C. K., \& Malheiros, W. (2007). Análise do perfil lipídico em escolares. Arquivos Brasileiros de Cardiologia, 89(2), 73-78.

Sheibani, H., Esmaeili, H., Tayefi, M., Saberi-Karimian, M., Darroudi, S., Mouhebati, M., \& Ghayour-Mobarhan, M. (2019). A comparison of body mass index and percent body fat as predictors of cardiovascular risk factors. Diabetes \& Metabolic Syndrome: Clinical Research \& Reviews, 13(1), 570-575.

Simão, A. F., Précoma, D. B., Andrade, J. P. D., Correa Filho, H., Saraiva, J. F. K., Oliveira, G. M. M., \& Souza, W. K. S. B. (2013). I Diretriz brasileira de prevenção cardiovascular. Arquivos brasileiros de cardiologia, 101(6), 1-63.

Vekic, J., Zeljkovic, A., Stefanovic, A., Jelic-Ivanovic, Z., \& Spasojevic-Kalimanovska, V. (2019). Obesity and dyslipidemia. Metabolism, 92, 71-81.

Vizentin, N. P., Cardoso, P. M. S., Maia, C. A. G., Alves, I. P., Aranha, G. L., \& Giannini, D. T. (2019). Dyslipidemia in adolescents seen in a university hospital in the city of Rio de Janeiro/Brazil: prevalence and association. Arquivos brasileiros de cardiologia, 112(2), 147-151.

Wang, H. H., Garruti, G., Liu, M., Portincasa, P., \& Wang, D. Q. H. (2017). Cholesterol and lipoprotein metabolism and atherosclerosis: recent advances in reverse cholesterol transport. Annals of hepatology, 16, S27-S42.

World Health Organization. (2019). World health statistics 2019: monitoring health for the SDGs, sustainable development goals. Geneva: WHO. https://apps.who.int/iris/bitstream/handle/10665/324835/9789241565707-eng.pdf?sequence=9\&isAllowed=y

World Health Organization. (2020). Obesity and overweight. Geneva: WHO. http:// www.who.int/en/news-room/fact-sheets/detail/obesity-and-overweight 